\title{
Elevation of intracellular calcium content in area CA1 of hippocampus is not directly correlated with the development of long-term potentiation
}

\author{
L. Siklós ${ }^{1}$, U. Kuhnt ${ }^{2}$, A. Mihály ${ }^{3}$ and F. Joó ${ }^{1}$ \\ 'Institute of Biophysics, Biological Research Center, Szeged (Hungary), ${ }^{2}$ Max-Planck-Institut für \\ biophysikalische Chemie, Göttingen (F.R.G.) and ${ }^{3}$ Department of Anatomy, Szent-Györgyi Medical School, \\ Szeged (Hungary)
}

(Received 3 July 1989; Revised version received 7 August 1989; Accepted 7 August 1989)

Key words: Long-term potentiation; D-2-Amino-5-phosphonovalerate; Calcium; Mitochondrium; In vitro; Hippocampus; Guinea pig

Electron microscopic localization of calcium-containing mitochondria in stratum radiatum of CAI of hippocampal slices was performed after (1) low-frequency stimulation, (2) high-frequency stimulation, and (3) blocking $N$-methyl-D-aspartate (NMDA) receptors during high-frequency stimulation. Dendritic mitochondria containing $\mathrm{Ca}$ deposits were found in a narrow band of stratum radiatum 280-350 $\mu \mathrm{m}$ distant from stratum pyramidale. Axonal mitochondria containing Ca deposits were evenly distributed in stratum radiatum. The total number of calcium containing-mitochondria was highest in long-term potentiated slices, and less in slices treated with APV; the lowest values were obtained with low-frequency stimulation.

High-frequency stimulation induces a persistent increase of synaptic efficacy in hippocampal neurones, termed long-term potentiation (LTP) [2]. Among the factors responsible for LTP the role of calcium ions $[1,15]$, and of $N$-methyl-D-aspartate (NMDA) receptors $[3,6]$ have been emphasized. Postsynaptic $\mathrm{Ca}^{2+}$ uptake has been shown to be coupled to the activation of NMDA receptors [5, 12], and the blockage of NMDA receptors by the selective antagonist $D$-2-amino-5-phosphonovalerate (APV) prevents the induction of LTP $[3,4]$. The aim of the present experiments, based on our previous studies $[10,13,14]$ was to reveal a relationship between the NMDA-mediated induction of LTP and mitochondrial Ca-accumulation.

The physiological protocol to induce and control LTP has been described earlier [10]. Briefly: transverse hippocampal slices $(400-500 \mu \mathrm{m})$ were prepared from right hippocampi of adult guinea pigs (450-600 g b.wt.) and incubated at room temperature in a medium containing in $\mathrm{mM}: \mathrm{NaCl} 124, \mathrm{KCl} 5, \mathrm{NaH}_{2} \mathrm{PO}_{4} 1.25, \mathrm{MgSO}_{4} 2$, $\mathrm{CaCl}_{2} 2, \mathrm{NaHCO}_{3} 26$, and glucose 10 and gassed by $\mathrm{O}_{2} / \mathrm{CO}_{2}(95 / 5 \%)(\mathrm{pH} 7.35-7.40)$.

Correspondence: L. Siklós, Max-Planck-Institut für biophysikalische Chemie, Abteilung Neurobiologie, D-3400 Göttingen, P.O. Box 2841, F.R.G. 

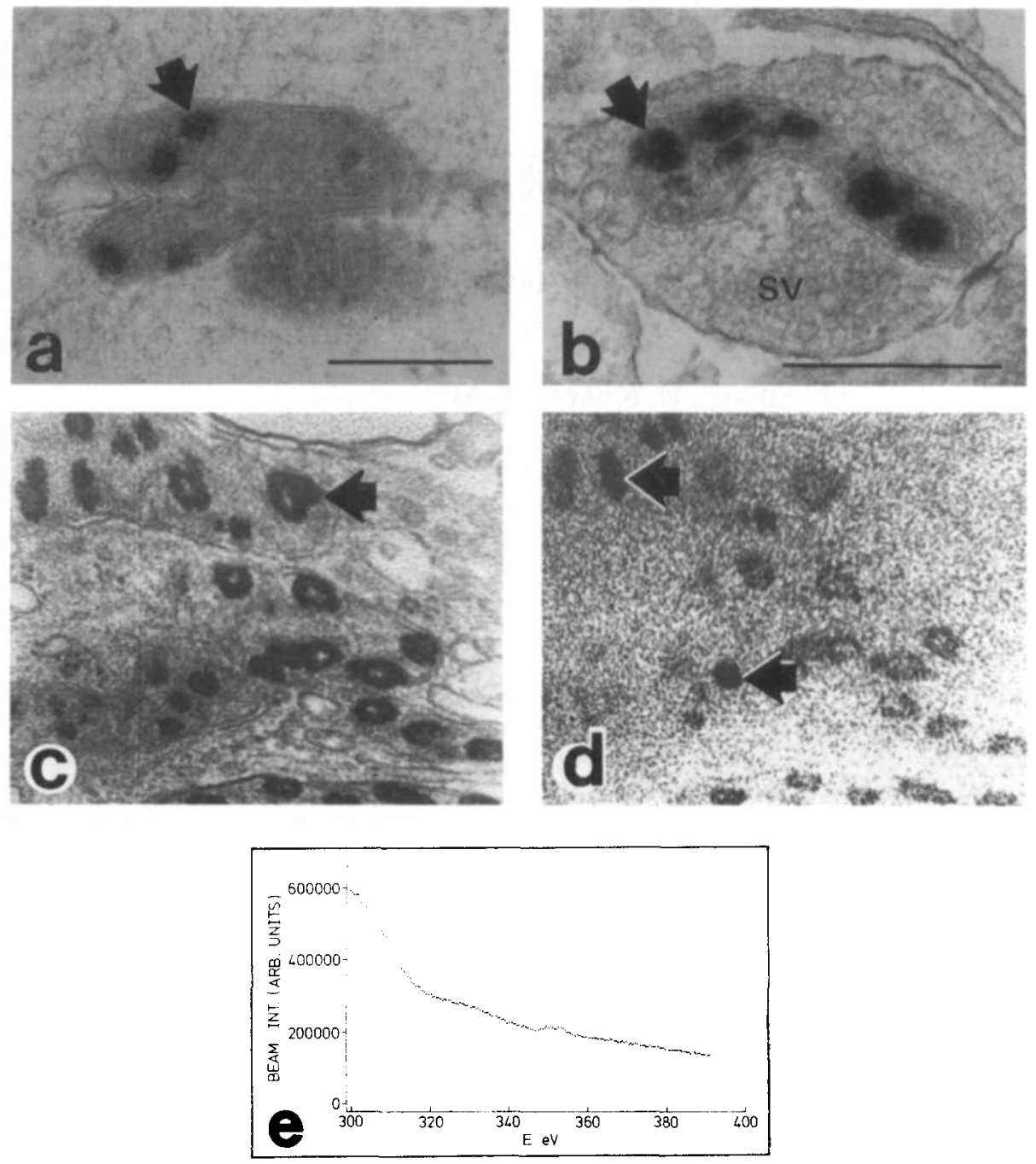

Fig. 1. Appearance and composition of EDDs. a, b: electron-dense deposits in mitochondria of dendritic (a) and axonal (b) compartments (arrows) as seen on conventional electron micrographs. sv: synaptic vesicles. Bar $=500 \mathrm{~nm}$. c: micrograph of mitochondrial EDDs obtained by inelastically scattered electrons in a ZEISS CEM electron microscope. The photograph has been taken from the screen of the computer and has been inverted for easier comparison with conventional pictures. The round shaped deposits are clearly recognizable (arrow). d: ESI picture of the same area shown on c. Recordings were made below and above the absorption edge of $\mathrm{Ca}$, then the images were subtracted from each other and thus result in the net Ca-distribution. The area showing high Ca-concentration (arrows) evidently correlates with the electron-dense deposits of c. e: electron energy loss spectrogram of a selected EDD. Presence of calcium in the material is evidenced by the peaks between 340 and $360 \mathrm{eV}$. The high background is a consequence of the relatively large section thickness. The vertical axis exhibits the beam intensity in arbitrary units. 
Single slices were then transferred to the recording chamber and kept at $35 \pm 1{ }^{\circ} \mathrm{C}$. The stimulating electrode was positioned in the Schaffer collateral/commissural pathway near the CA3/CAl border. Control stimuli were given at $0.125 \mathrm{~Hz}$, high-frequency stimulation (10 trains of 20 stimuli at $100 \mathrm{~Hz}$ in $8 \mathrm{~s}$ intervals) was applied at the same stimulus intensity. Recording electrodes were positioned in stratum pyramidale (str.pyr.) and in stratum radiatum (str.rad.); control responses were stored on a laboratory computer. APV (final concentration $200 \mu \mathrm{M}$ ) was applied 30 min before and switched off $5 \mathrm{~min}$ after high-frequency stimulation. After high-frequency stimulation slices were stimulated for $1.5 \mathrm{~h}$ at low frequency. Slices were then transferred in a fixative containing $\mathrm{CaCl}_{2}(10 \mathrm{mM})$ and processed for electron microscopy according to the calcium inclusion procedure [13]. Sections were placed onto single-slot grids, stained with lead citrate, and traced at $6000 \times$ instrumental magnification. The distribution of axonal and dendritic mitochondria containing electron dense deposits (EDDs) was mapped in str.rad. of CAl (Fig. 2) between the stimulating and recording electrodes, by using a computer fitted to the electron microscope (EM) to read and store the position of the specimen during examination.

The calcium inclusion procedure [13] yielded round shaped uniform EDDs in mitochondria (Fig. la, b). The calcium content of the EDD's was proven by electron spectroscopic imaging (ESI) and electron energy loss spectroscopy (EELS) (Fig. 1c-e). During analysis of a series of differently treated slices, sections with the same thickness were used, since the number of recognized EDD-containing mitochondria was

\section{TABLE I}

\section{NUMBER OF EDD-CONTAINING MITOCHONDRIA}

The total number of EDD-containing mitochondria depends on the thickness of the section. CONT, low frequency stimulation; APV, treatment with AVP during high-frequency stimulation; LTP, LTP induced by high-frequency stimulation; $n$, absolute number of counted EDD-containing mitochondria in the respective compartment; axonal, dendritic, investigated compartments; total, total number of counts in a series; $\%$, absolute values given as percentage of the total number of EDD-containing mitochondria in the series.

\begin{tabular}{|c|c|c|c|c|c|c|c|}
\hline \multirow{2}{*}{$\begin{array}{l}\text { Section } \\
\text { thickness }\end{array}$} & \multirow[t]{2}{*}{ Treatment } & \multicolumn{2}{|c|}{ Axonal } & \multicolumn{2}{|c|}{ Dendritic } & \multicolumn{2}{|l|}{ Sum } \\
\hline & & $n$ & $\%$ & $n$ & $\%$ & $n$ & $\%$ \\
\hline Silvergray & CONT & 59 & 11 & 15 & 3 & 74 & 14 \\
\hline \multirow[t]{2}{*}{$50-70 \mathrm{~nm}$} & APV & 103 & 19 & 50 & 9 & 153 & 28 \\
\hline & LTP & 246 & 45 & 73 & 13 & 319 & 58 \\
\hline Total: & & & & & & 546 & 100 \\
\hline Goldyellow & CONT & 157 & 11 & 1 & $<1$ & 158 & 11 \\
\hline \multirow[t]{2}{*}{$90-110 \mathrm{~nm}$} & APV & 272 & 20 & 94 & 7 & 366 & 27 \\
\hline & LTP & 728 & 52 & 133 & 10 & 861 & 62 \\
\hline Total & & & & & & 1385 & 100 \\
\hline
\end{tabular}


affected by the section thickness (Table I). The total number and the distribution of mitochondria containing EDD's changed according to the physiological manipulation. The highest concentration of loaded mitochondria was obtained after induction of LTP. The amount dropped to about half if the NMDA receptors were blocked during high-frequency stimulation. In low-frequency-stimulated slices the total number of loaded mitochondria dropped further (Fig. 2, Table I). The distribution of loaded mitochondria in the axonal and in the dendritic compartment differed strongly. Dendritic EDD-loaded mitochondria were concentrated in a narrow band. This band run parallel to str.pyr. at a distance of $280-350 \mu \mathrm{m}$ and stretched across the area investigated (Fig. 2). The distance of this band coincided in all cases with the distance of the stimulation site from str.pyr. Highest concentrations of dendritic EDDs were

A

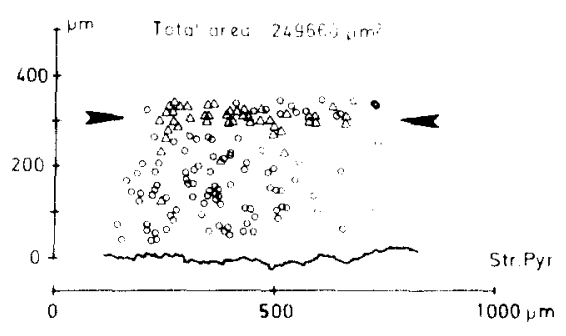

B

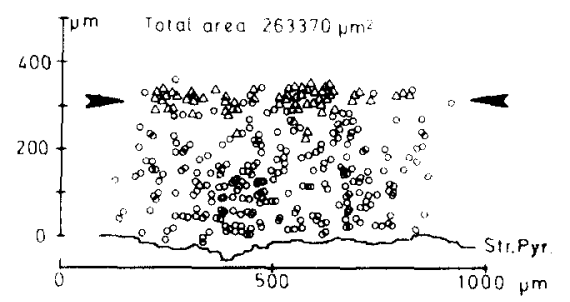

C
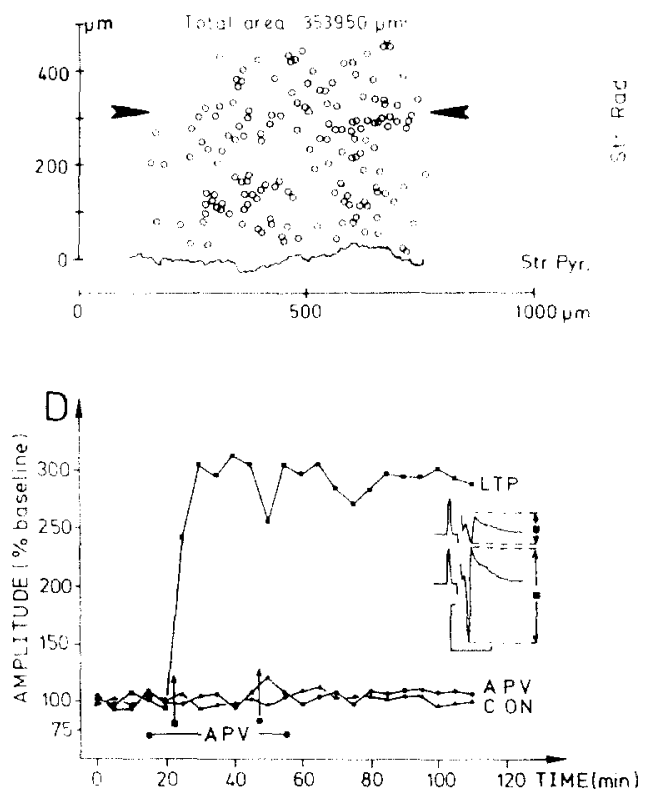

Fig. 2. Distribution of EDDs containing mitochondria in str.rad. A C: computer plots of the spatial distribution of loaded mitochondria in the presynaptic (open circle) and the postsynaptic compartments (open triangle). The middle of str.pyr. is marked by the continuous line, str.rad. extends in the vertical direction, calibration as indicated. The total investigated area of the section is indicated at the top of the diagrams. A: section of an APV-treated slice (see D). Loaded mitochondria in the postsynaptic compartment are concentrated in a narrow band, indicated by arrows. B: section of a slice after successful induction of LTP (see D). $C$ : section of a low-frequency-stimulated slice (see D). Only a single loaded mitochondria is seen in the postsynaptic compartment. D: amplitude time course of averaged evoked potentials $(n=10)$ for the different conditions (A C). Horizontal axis: time in minutes, vertical axis: amlitude given as $\%$ of baseline (peak-to-peak amplitude measurement of the population spike as shown in inset). The mean of measurement 15 is taken as baseline (100\%). Time of APV application is indicated by a bar. Time of high-frequency stimulation is indicated by arrows with filled circle and filled square for APV-treated and LTP slices, respectively. 
encountered in slices displaying LTP, followed by APV-treated material, whereas in low-frequency-stimulated material only few EDD-loaded mitochondria were found. In contrast, axonal EDD-loaded mitochondria were spread diffusely over the whole depth of str.rad. Their number showed a similar dependance on the experimental conditions as was found for the dendritic compartment. (Table I, Fig. 2).

Mitochondria possess the capability to regulate the free cytoplasmic calcium concentration by storing excess ions in bound form [11]. We interprete the observed increase of loaded mitochondria as the result of an excessive influx of calcium, due to high frequency stimulation. The postsynaptic aggregation of loaded mitochondria points to spatially restricted changes of the internal Ca-concentration [5]. The largest numbers of loaded mitochondria were counted after successful induction of LTP. It is tempting to interpret this finding as a consequence of a strong local Ca-influx following the opening of NMDA dependent Ca-channels, as proposed by Collingridge et al. [3, 4]. The treatment with a selective NMDA antagonist which suppressed the induction of LTP, led to a reduction of the $\mathrm{Ca}$ accumulation, but it was clearly elevated if compared with the results from control material. The most obvious interpretation is an influx of calcium ions through other route(s) which, alone, are not sufficient to induce LTP. Our conclusion that a local increase of the intracellular Ca-concentration is not sufficient to induce LTP is supported by fluorometric measurements of calcium kinetics in hippocampal slices [9] and in isolated CA1 pyramidal neurones [5]. The local redistribution of differently bound calcium cannot be excluded, but seems unlikely considering the above mentioned reports. The overall localization of calcium loaded mitochondria in the presynaptic compartment can be explained by the course of axons [8]. The dependance of their number on the experimental procedure, however, might be caused by postsynaptic 'trophic' effects on presynaptic elements [7], possibly transmitted by poorly understood secretion/diffusion mechanisms.

L.S. and A.M. were supported by the Max-Planck-Society and by the Alexander von Humboldt Foundation, respectively. The authors wish to thank Ms S. Lausmann and Ms E. Nicksch for their skilful technical assistance. The authors are greatly indebted to Dr R. Bauer, ZEISS Application Laboratory, Oberkochen, for performing the ESI and EELS measurements.

1 Baimbridge, K.G. and Miller, J.J., Calcium uptake and retention during long-term potentiation of neuronal activity in the rat hippocampal slice preparation, Brain Res., 221 (1981) 299-305.

2 Bliss, T.V.P. and Lømo, T., Long-lasting potentiation of synaptic transmission in the dentate area of the anaesthetized rabbit following stimulation of the perforant path, J. Physiol. (Lond.), 232 (1973) $331-356$.

3 Collingridge, G.L., and Bliss, T.V.P., NMDA receptors - their role in long-term potentiation, Trends Neurosci., 10 (1987) 288-293.

4 Collingridge, G.L., Kehl, S.J. and McLennan, H., Excitatory amino acids in synaptic transmission in the Schaffer collateral-commissural pathway of the hippocampus, J. Physiol. (Lond.), 334 (1983) 33.46.

5 Connor, J.A., Wadman, W.J., Hockberger, P.E. and Wong, R.K.S., Sustained dendritic gradients of $\mathrm{Ca}^{2+}$ induced by excitatory amino acids in CAl hippocampal neurons, Science, 240 (1988) 649-653. 
6 Cotman, C.W., Monaghan, D.T. and Ganong, A.H., Excitatory amino acid neurotransmission: NMDA receptors and Hebb-type synaptic plasticity. Annu. Rev. Neurosci., 11 (1988) $61-80$.

7 Gage, F. and Björklund, A., Trophic and growth-regulating mechanisms in the central nervous system monitored by intracerebral neural transplants. In G. Bock and M. O'Connor (Eds.). Selective Neuronal Death, Wiley, Chichester, 1987, pp. 143155.

8 Hjorth-Simonsen. A. Some intrinsic connections of hippocampus in the rat: an experimental analysis, J. Comp. Neurol., 147 (1973) 145162.

9 Kato, H., Miyakawa, H.. Ogure, A. and Kudo, Y., Cytoplasmic $\mathrm{Ca}^{2+}$ changes in reference to longterm potentiation in the hippocampal slice. In H.L. Haas and G. Buzsáki (Eds.), Synaptic Plasticity in the Hippocampus, Springer, Berlin. 1988, pp. 4648

10 Kuhnı, U., Mihály, A.. Siklós, L. and Joó, F., Increased retention of calcium in the dendrites of longterm potentiated CAl neurons of the hippocampal slice. A combined electrophysiological and electron histochemical study, Histochemistry, 90 (1988) 185193.

11 Lehninger, A.L., Reynafarje, B., Vercesi, A. and Tew. W.P.. Transport and accumulation of calcium in mitochondria, Ann. N.Y. Acad. Sci., 307 (1978) 160-173.

12 MacDermott, A.B., Mayer, M.L., Westbrook, G.H., Smith, S.J. and Barker, J.L., NMDA-receptor activation increases cytoplasmic calcium concentration in cultured spinal cord neurones, Nature (Lond.), 321 (1986) 519522

13 Mihály, A., Kuhnt, U.. Siklós. L. and Joo, F., Semiquantitative evaluation of histochemically detectable calcium binding in mammalian brain slices, J. Neurosci. Methods, 20 (1987) 283.293.

14 Siklos, L., Kuhnt, U., Joó. F. and Mihály, A., Electron histochemical localization of calcium in (A) of hippocampal slices following high frequency stimulation: effects of excitatory amino acid antagonists. In H.L. Haas and G. Buzsáki (Eds.), Synaptic Plasticity in the Hippocampus, Springer, Berlin, 1988. pp. 49.52.

15 Wigström, H., Swann. J.W. and Andersen, P., Calcium dependency of synaptic long-lasting potentiation in the hippocampal slice, Acta Physiol. Scand., 105 (1979) 126 128. 